\title{
Microstructure Study of Carbon Nanocages Consisting of Graphene Oxide Grafted with Single Gold Nanoparticles by Application of HAADF Contrast Imaging
}

\author{
Snejana Bakardjieva ${ }^{1}$, Mario Bakardjiev ${ }^{1}$, Robert Klie ${ }^{2}$ and Chanhui Wang ${ }^{2}$ \\ 1. Institute of Inorganic Chemistry of the CAS, Republic. \\ 2. University of Illinois at Chicago, Department of Physics, Chicago, USA.
}

Microstructural investigation of graphene based materials by using High Angle Annular Dark Field (HAADF) imaging in aberration corrected STEM microscopy techniques is very challenging due to strong image contrast resulting from the high contrast density. The HAADF imaging advantages for observation of grain fragmentation, nucleation of recrystallized grains and precipitation on second phase particles. By using STEM-HAADF imaging, various microstructural features have been imaged, such as nanoscale subgrain structure and recrystallization nucleation even from the thicker region of the highly thin materials. HAADF images are formed by incoherent thermal diffuse scattering (TDS) electrons, utilizing a large inner angle detector [1]. Furthermore, in incoherent imaging, each atom independently contributes intensity to the resulting Z-contrast image. Many studies demonstrated that graphene oxide (GO) monolayers can be thought as a 2D building block for carbon nanostructures of other dimensionalities. It can be wrapped into 0D buckyballs, rolled into 1D nanotubes, or stacked into 3D graphite. Recently, Li [2] demonstrated transformation of flat GO sheets into fullerene cages. In this work, for the first time, HAADF-STEM methodology has been applied to observe the morphology of gold nanoparticles (Au NPs) anchored on carbon nanocages formed by curved nono-sized GO shells. Nanocages with diameters ranging between 10 and $60 \mathrm{~nm}$ were attained via an interacation reaction between GO sheets and Au NPs [3]. The hybrid structure was prepared by doping of Sigma-Aldrich sertificated GO with Au AAS TraceCERT solution in the presence of urea.The photocatalytic activity of as prepared hybrid was assessed by the photocatalytic decolorization of Rhodamine B dye under UV/Vis irradiation.

An aberration corrected STEM is an ideal tool for atomic scale studies of the metal-GO system, especially for suspended GO. Characterization of structures at atomic resolution was performed by UIC aberration-corrected ARM200CF microscope by using ABF/LAADF/HAADF detectors at 80kV. The ABF/STEM images in Fig. 1a, low magnification and Fig. 1b, high mannification, reveal carbon nanocages composed of curved carbon structures consisting of graphene layers. The ABF/STEM image reported in Fig. 1c-d shows atomic columns of Au NPs capped on the graphene layers. The HAADF image acquired from same region with camera length $80 \mathrm{~mm}$ showing Au grains refinement and bright intensity from the Au NPs precipitates, see Fig. 2(a-d). HAADF imaging technique can be used to find $\mathrm{Au}$ NPs particle size distribution onto the surface of GO as it presented in Fig. 2(b-d). The HAADF study reveals the direct interface between Au NPs and GO sheet, which can be manipulated the electronic structure and can be shifted the absorbance in the visible region due to the surface plasmon resonance absorption of Au NPs. High magnification image (Fig. 2d) confirmed that Au NPs effectively separated the GO sheets and in this manner could be preventing sheets aggregation. The binding of $\mathrm{Au}$ NPs occurred at the edges of carbon nanocages ( HAADF/STEM images in Fig. 2c-d). The size of the $\mathrm{Au}$ NPs was about $3 \mathrm{~nm}$, which is very desirable to provide an optical enhancement effect by surface plasmonic resonance in the full visible range. We are expecting that plasmonic photocatalyst systems consist of GO sheets decorated with Au NPs will give rise to an extraordinary modification to the nanoplasmonic properties of the resultant hybrid. 
In our study, the advantage of HAADF STEM imaging for identifying Au NPs particles can be clearly observed. The morphological and structural characterizations evidence that the Au NPs can be efficiently decorated on the GO. The GO sheets could serve as support material to stabilize Au NPs . Well-dispersed $\mathrm{Au}$ NPs could attract excited electrons under UV/Vis light and better up the conductivity of carbon nanocages. The rate constants for decolorization of Rhodamine B dye by using as prepared hybrid are 0.7391 and $0.5901 \mathrm{~min}^{-1}$ for UV and Vis irradiation, respectively. Carbon nanocages consisting of GO grafted with single Au NPs can provide a suitable catalytic platform for environmental pollutants [4].

\section{References:}

[1] Cowley J., Ultramicroscopy 2 (1977), p.16.

[2] Z. Li, Chem.Mater. 19 (2007), p. 3349.

[3] S.Ge et al, Bioelectr. 31 (2012), p.49.

[4] The authors acknowledge Prof. R. Nikolova from IPC BAS for the catalytic experiment.

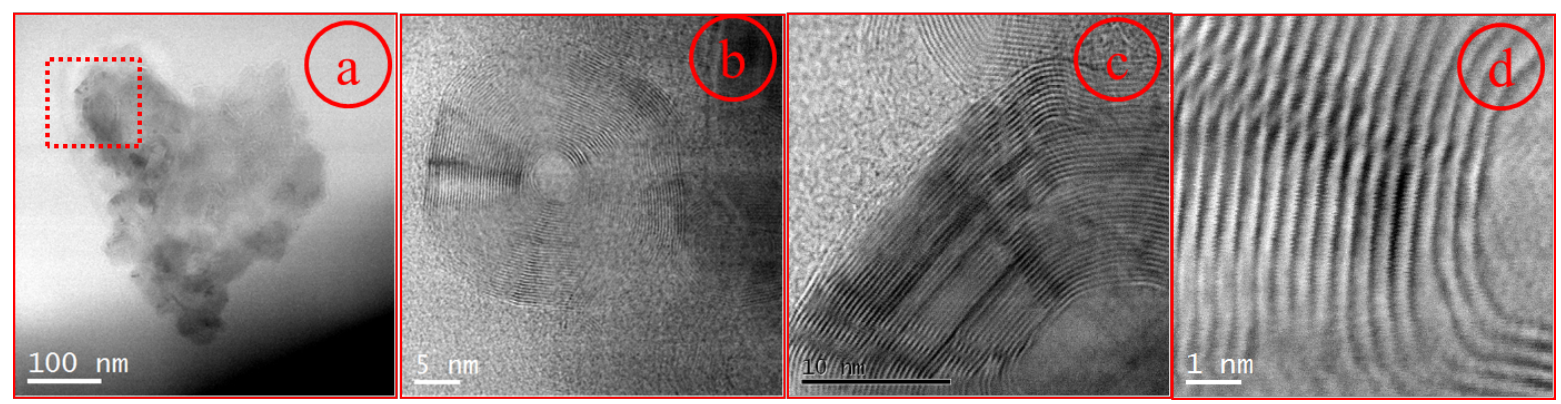

Figure 1. The conventional TEM bright field image showing morphology of carbon nanocages consisting of GO grafted with single Au NPs (a-b) Low magnification, (c-d) High magnification revealed layered structure of atomically thin GO with Au NPs.
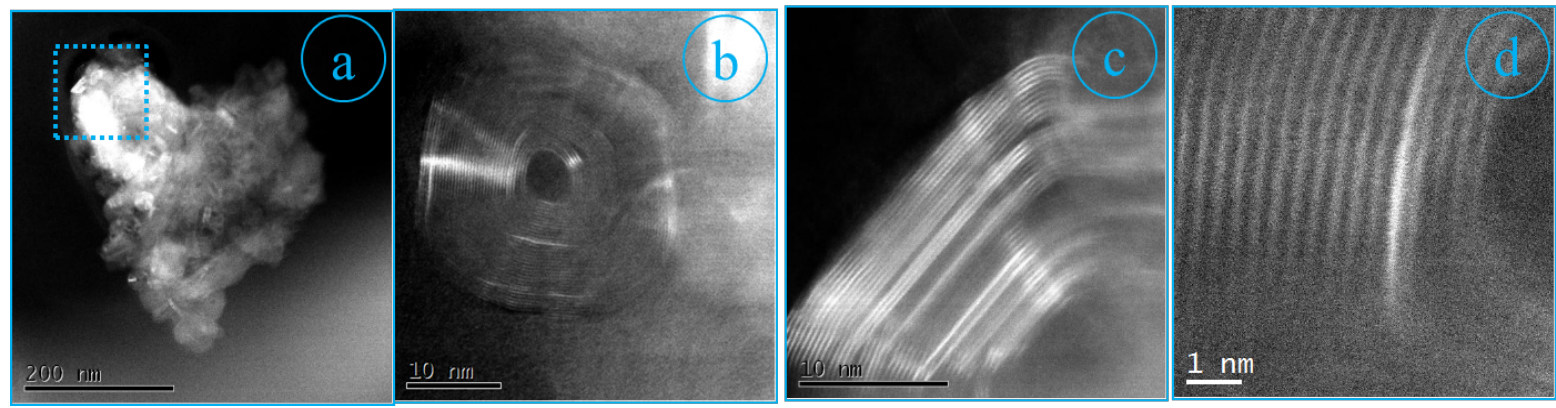

Figure 2. The HAADF image acquired from same region with camera length $80 \mathrm{~mm}$ showing Au grains refinement and bright intensity from the Au NPs (a-d) HAADF imaging technique can be used to find $\mathrm{Au}$ NPs particle size distribution onto the surface of GO. 